\title{
Experiencia de 100 intubaciones y 100 traqueotomías en asfixia por Crup diftérico
}

\author{
Publicado en Revista Chilena de Pediatría 1938
}

\author{
Autores: CESAR IZZO \\ Comentario: LUISA SCHONHAUT B. ${ }^{1}$, STEFAN GUILOFF P. ${ }^{2}$ \\ 1. Departamento de Pediatría, Facultad de Medicina Clínica Alemana - Universidad del Desarrollo. \\ 2. Interno de Medicina. Universidad Mayor.
}

Key words: Tracheotomy, tracheostomy, intubation, diphtheria, pediatric history.

Palabras clave: Intubación, traqueostomía, traqueotomía, difteria, historia pediatría.

\section{Contexto histórico}

"Pocas veces un cirujano se siente más tranquilo y más seguro de ser rápidamente útil, que con un aparato de intubación en la mano, ante un pequeño diftérico que se asfixia”.

(Izzo 1938).

Hasta mediados del siglo XX los indicadores de salud infantil eran desoladores, sumado al flagelo de la desnutrición y las incurables enfermedades infectocontagiosas, devastadoras epidemias azotaban al país y se llevaban consigo la vida de muchos "angelitos". En este contexto se fundaron los primeros hospitales pediátricos, cuya función primordial era dar hospedaje y albergue a los enfermos y desamparados ${ }^{2}$; como cabría esperar, la mortalidad intrahospitalaria era elevadísima, dado que más de la mitad de los niños "ingresan en tan malas condiciones generales, con enfermedades graves, algunas incurables..."(Baeza Goñi 1938) y las alternativas terapéuticas eran limitadas ${ }^{2}$. En aquellos años las unidades de cuidados intensivos y los ventiladores mecánicos eran sueños lejanos, los recién descubiertos antibióticos se encontraban en fase experimental y la universalización de las vacunas era una utopía.

La difteria era una de las primeras causas de morbimortalidad infantil en países industrializados; en nuestro país anualmente se notificaban entre 600 y 1.000 casos, con una tasa de letalidad del orden del 20 a $30 \%{ }^{3}$. La elevada mortalidad se debía generalmente a su presentación "maligna" o a la obstrucción laríngea o croup, que culminaba en una cuadro de sofocación o asfixia, de ahí que se le conociera como "Morbus Strangulatorius" o "garrotillo"4,5.

En la primera mitad del Siglo XIX se comenzó a indicar la traqueotomía para el manejo de las obstrucciones de la vía aérea superior ${ }^{6}$; si bien este método parecía prometedor, más de la mitad de los sometidos a este procedimiento fallecía?. "la introducción de la 
traqueotomía ha sido de gran mérito para la Humanidad, pero será un mérito mucho mayor cuando aprendamos a evitarla", esta frase "encerraba profecía" dado los "descorazonadores" resultados del procedimiento, hace un poco más de 75 años ${ }^{8}$.

La investigación del Dr. Izzo, publicada el año 1938 y reproducida en este número, corresponde al primer ensayo clínico reportado en nuestra revista. Este ensayo, fundamentado en experiencias internacionales, comenzó como un sueño algunos años antes ${ }^{8}$. En su investigación, Izzo comparó la sobrevida de niños que fueron intubados con una cohorte histórica de niños traqueostomizados "hasta el 4 de abril del año 1934 no hacíamos sino traqueotomías y desde esa fecha, exclusivamente intubaciones, cualquiera que fuese el tipo de asfixia por difteria". Como se puede desprender, la asignación de pacientes no fue aleatorizada, ciega, ni controlada, sin embargo, logró demostrar que la intubación reducía la mortalidad de 62 a 26\%, y con un mínimo de complicaciones. Según refiriera el autor, "En nuestro caso, el centenar de traqueotomías y el centenar de intubaciones es perfectamente comparable" puesto que el genio epidémico y las posibilidades de tratamiento no habían variado en los años del estudio.

Sin duda, las observaciones de Cesar Izzo significaron un enorme avance, un éxito tal, que decidió reportar los resultados preliminares, de modo que su experiencia pudiese ser reproducida en otros centros del país. "...los brillantes resultados obtenidos con la intubación, nos obligan a presentar 51 casos que poseemos, porque nos sentiríamos culpables de los accidentes y fracasos obtenidos con nuevas traqueotomías al no hacer desde ya, todo lo posible, para que en nuestro país se reemplace la traqueotomía sistemática por la intubación".

\section{Artículo de Cesar Izzo}

Hace dos años, presentamos a esta Sociedad un pequeño trabajo, exponiendo los resultados obtenidos con la intubación en el crup diftérico. Habíamos efectuado en ese entonces
40 intubaciones y alrededor de 105 traqueotomías, cifras dispares, pero en que ya resaltaba la gran ventaja de la intubación. Es por eso que nos adelantamos a publicar los resultados para iniciar una abierta campaña en favor de la intubación y tranquilizar nuestra conciencia, que se encontraba inquieta, al observar la alta mortalidad de la traqueotomía, pudiendo ser reemplazada casi totalmente por otro método tan simple.

Sin embargo, prometimos que en una fecha próxima expondríamos el resultado de cifras más altas y en igualdad de condiciones.

Hoy venimos a cumplir ese compromiso.

Una estadística a menudo merece reparos e inspira desconfianza, ya que se ha dicho "que es una mujer coqueta que se inclina al más galante"; sin embargo, algunas veces reúnen condiciones dignas de merecernos absoluta fe. En nuestro caso, el centenar de traqueotomías y el centenar de intubaciones es perfectamente comparable, por las siguientes razones:

El genio epidémico de la difteria se ha mantenido el mismo en nuestro país desde hace más de 6 años, siendo raras las difterias malignas, y presentando la mayor gravedad, por lo tardío del tratamiento por la dejación de nuestra clientela hospitalaria en acudir precozmente al médico.

Por otra parte, no se ha hecho elección alguna de los casos para efectuar una u otra intervención, sino que hasta el 4 de abril del año 1934 no hacíamos sino traqueotomías y desde esa fecha, exclusivamente intubaciones, cualquiera que fuese el tipo de asfixia por difteria.

En estas circunstancias, debemos manifestar que las traqueotomías siempre estuvieron a cargo de personal experimentado, en cambio, la intubación, como nuevo método para nuestro Servicio, hubo de soportar el aprendizaje de más de una docena de médicos.

En cuanto al tratamiento general del crup, ha permanecido sensiblemente el mismo: dosis altas de suero antidiftérico, dando la mayor importancia a la dosis primera; antiespasmódicos, belladona, atropina; sedantes: cloral, sedol, etc.

Con estas consideraciones generales, podemos concluir que ambas centenas de casos son perfectamente comparables y si alguna des- 
ventaja hay, es para la intubación.

Veamos lo que nos dice el estudio comparativo:

\begin{tabular}{|c|c|c|c|c|c|c|}
\hline \multirow[b]{2}{*}{ Edad } & \multicolumn{3}{|c|}{ Traqueotomías } & \multicolumn{3}{|c|}{ Intubación } \\
\hline & $\mathbf{n}$ & Fallecidos & $\%$ & $\mathbf{n}$ & Fallecidos & $\%$ \\
\hline 0 a 1 & 9 & 7 & 77,7 & 6 & 2 & 33,3 \\
\hline 1 a 2 & 20 & 15 & 75 & 17 & 4 & 23,5 \\
\hline 2 a 3 & 14 & 10 & 71,4 & 20 & 3 & 15 \\
\hline 3 a 4 & 15 & 10 & 66,6 & 19 & 7 & 36,8 \\
\hline 4 a 5 & 14 & 7 & 50 & 10 & 2 & 20 \\
\hline 5 a 6 & 7 & 3 & 42 & 6 & 1 & 16,6 \\
\hline 6 a 7 & 7 & 4 & 57,1 & 8 & 5 & 62,5 \\
\hline 7 a 8 & 5 & 2 & 40 & 4 & 0 & 0 \\
\hline 8 a 9 & 4 & 2 & 50 & 6 & 0 & 0 \\
\hline 9 a 10 & 2 & 1 & 50 & 3 & 2 & 66,6 \\
\hline 10 a14 & 3 & 1 & 33,3 & 1 & 0 & 0 \\
\hline & 100 & 62 & & 100 & 26 & \\
\hline
\end{tabular}

Se puede observar la mayor frecuencia del crup entre 1 y 4 años, época en que la glotis es proporcionalmente más pequeña. Época también en que la traqueotomía es más dificultosa por ser cartilaginosa y blanda la tráquea, ofreciendo menos puntos de reparo al cirujano y a la vez que son más frecuentes los síncopes al abrir la tráquea. De ahí que aparezcan las más altas mortalidades de la traqueotomía, 77,7\%, $75 \%, 71,4 \%, 66,6 \%$.

La mortalidad total es, como puede verse en el cuadro, de $62 \%$ para los traqueotomizados contra $26 \%$ de los intubados, o sea que la mortalidad hemos logrado disminuirla en más del 50\%. Dicho de otra forma, mientras de 10 traqueotomizados se nos morían 6, de 10 intubados sólo fallecen 2 .

Esta es la mortalidad global en la que entran como causa la difteria tóxica, el acto operatorio, la bronconeumonía, etc.

La mortalidad operatoria, o sea, la que se produce durante la ejecución de la traqueotomía o intubación, da diferencia muy notable, es de $11 \%$ para la traqueotomía y sólo $1 \%$ para la intubación.

Nada hay más desagradable para el cirujano que al efectuar una traqueotomía, a veces al iniciar la intervención, aparezca el temido síncope respiratorio que a veces pasa y otras dura la eternidad. Hemos perdido así 11 enfermos de 100 operados, cifra desconsoladora, difícil de disminuir aún con técnica acabada. En cambio, con que satisfacción se efectúa la intubación, que no tiene ningún riesgo operatorio si se considera su mortalidad de $1 \%$ y aún ese caso fallecido, se debió a que existiendo una anomalía, un divertículo traqueal que se comprobó en la autopsia, el tubo siguió una ruta equivocada.

Es tan excepcional esta circunstancia que bien podríamos considerar "cero" la mortalidad operatoria de la intubación. Y es esto lo que me ha hecho decir en otras oportunidades, que "pocas veces un cirujano se siente más tranquilo y más seguro de ser rápidamente útil, que con un aparato de intubación en la mano, ante un pequeño diftérico que se asfixia".

\section{Complicaciones de la intubación}

La más frecuente es la bronconeumonía, pero es sumamente difícil precisar en un niño en crup franco si existe un foco de bronconeumonía y, además, hay que actuar rápido y una vez hecha la traqueotomía o intubación, no se pueden precisar los ruidos pulmonares, quedando sólo útil la percusión y los Rayos $\mathrm{X}$, siendo la primera inconstante y lo segundo poco práctico, dada la gravedad del enfermo.

Es por esto imposible decir cuántas veces la bronconeumonía es complicación de la intervención y cuantas veces venía ya en incubación o recientemente declarada.

De ahí que hemos optado por considerar el total de casos en que posteriormente apareció una bronconeumonía y la hemos encontrado en 28 de los 100 traqueotomizados y sólo en 10 de los 100 intubados. Es lógico, entonces, pensar que la intubación no es ajena a esta notable disminución de la complicación pulmonar. Y se explica fácilmente ya que usando las vías naturales, el aire llega a los pulmones en condiciones normales, húmedo y filtrado y no impuro y frío como sucede al pasar directamente a la tráquea por la cánula de traqueotomía.

La movilización de membranas, empujadas al colocar el tubo, es otra complicación que temíamos. Efectivamente, al introducir el tubo, éste separa las cuerdas vocales y la membrana puede quedar en el lumen de la laringe y ser empujada hacia abajo, a la vez que obturar el tubo. En cuatro casos nos ha ocurrido esto y 
hemos procedido no retirando el tubo, que parecería lo más lógico, sino que con toda calma continuar su introducción y entonces el tubo mismo perfora la membrana o la echa a un lado, estableciéndose la respiración.

La expulsión del tubo es otro temor infundado. Tenemos un caso actualmente en el Servicio que se reintubó 7 veces, sin molestias posteriores. Otro caso de 6 reintubaciones, y 2 casos con 5 reintubaciones. El examen laríngeo en 3 de ellos no revela lesión de decúbito alguna. Los otros 3 no se han podido examinar posteriormente. En total, 22 niños han debido ser reintubados; las causas han sido las siguientes: 6 han sido accesos de Coqueluche en niños con Difteria y Coqueluche; 4 por la tos de bronconeumonía; 3 por obstrucción completa del tubo con membranas; 4 por obstrucción parcial; 2 por arcadas voluntarias; 1 por tracción sobre el hilo (en la época en que dejábamos el hilo), y 2 por causas que no hemos podido precisar.

Se deduce, pues, que de 5 niños, uno debe ser reintubado una o más veces, pero la reintubación es sin peligro alguno y aún más sencilla que la intubación primera, ya que el primer tubo ha servido de dilatador discreto y facilita la reintubación. Por lo demás, una vez expulsado el tubo, la asfixia no sobreviene de inmediato, pudiendo a veces no ser necesaria la reintubación, aún cuando el tubo haya permanecido pocas horas en la laringe, todo depende de la mayor o menor cantidad de membranas que se eliminen durante la permanencia del tubo.

El fantasma de la reintubación, pues, no es tal.

La caída del tubo en el esófago es otro pequeño incidente que nos ha ocurrido 4 veces: una vez porque, al saltar con la tos, el niño lo deglutió; 2 veces por técnica defectuosa cuando iniciábamos el aprendizaje de la intubación, y la cuarta vez, sin poder precisar la causa. Seguramente expulsión por la tos y deglución.

En todos estos casos, el tubo se ha eliminado antes de las $48 \mathrm{~h}$, siguiendo el tractus intestinal, sin tropiezo alguno, ya que su forma alargada con todos sus bordes romos facilita el deslizamiento.

El niño Tubado, o sea el que por sufrir acostumbramiento al tubo, hayamos tenido que recurrir a la traqueotomía, no lo hemos visto nunca, a pesar que en algunos casos de niños neuropáticos con factores espasmódicos agregados, hemos tenido que dejar el tubo hasta 18 días. Por lo general, a las $72 \mathrm{~h}$, tiempo de elección, hemos retirado el tubo sin inconveniente.

La difteria descendente nos produce aún una ligera inquietud, pues la intubación es impotente ante ella, ya que la asfixia se produce en los bronquiolos, pero aún la traqueotomía, como se comprende, no puede descargar los bronquiolos. Sin embargo, como la brecha de esta última queda más cerca de la bifurcación bronquial, por la herida operatoria se logra, a veces, extraer largos moldes de membranas que alivian grandemente al enfermo.

Nosotros hemos continuado haciendo la intubación en estos casos, ya que si hubiera necesidad de traqueotomía, la ejecución de ésta se facilita grandemente, estando el tubo en la laringe, ya que facilita momentáneamente la respiración durante la ejecución de la traqueotomía, a la vez que sirve de magnífico reparo para encontrar rápidamente la tráquea.

Respecto a la técnica, no hemos hecho ninguna variación a la que hemos descrito anteriormente en diversos artículos. La anestesia local la hemos abandonado completamente, por innecesaria y la extracción del tubo la hacemos siempre por las maniobras externas que hemos descrito, teniendo que recurrir sólo excepcionalmente al extractor.

Con esta experiencia adquirida, mantenemos, como se comprende, nuestra convicción de la necesidad de ir cuanto antes al reemplazado de la traqueotomía sistemática por la intubación y nos es particularmente grato observar que nuestro esfuerzo por propagar la intubación, ha salido en nuestro país de los límites de la capital, pues ella se está empleando en algunas provincias del norte y del sur por colegas cuya correspondencia francamente optimista, nos halaga sobremanera. Sea para ellos los resultados de esta experiencia que hoy publico, otro estímulo que les haga reforzar el convencimiento que ya tienen de la necesidad de propagar la intubación en el crup diftérico, dejando la traqueotomía para casos absolutamente excepcionales.

En el año 1865, el gran cirujano Gageine, descorazonado por los resultados de la tra- 
queotomía, terminaba una de sus clases diciendo: "la introducción de la traqueotomía en la cirugía del crup diftérico, ha sido un gran mérito para la Humanidad, pero será un mérito mucho mayor cuando aprendamos a evitarla". Esta sabia frase encerraba profecía. Y hoy con el empleo de la intubación, estamos viviendo la realidad de esa profecía.

\section{Comentario del artículo de Cesar Izzo}

Hasta mediados del siglo XX en que se instauró la vacunación universal, la difteria era una entidad nosológica altamente prevalente y letal, que aparecía en forma de brotes cada 8 a 9 años 9 . Publicaciones de la época consideraban que la enfermedad en Chile era de poca gravedad, dado que la tasa de mortalidad, del orden de 6 por 100.000 habitantes, era inferior a los reportes internacionales "aventajando en varios aspectos a países de civilización más antigua y más extensa."(Mobarec 1935) ${ }^{10}$. No obstante, la difteria daba cuenta del 9\% del total de ingresos pediátricos y cerca del $5 \%$ era de curso maligno ${ }^{11,12}$. Se atribuía la gravedad de la presentación fundamentalmente a "lo tardío del tratamiento, por la dejación de nuestra clientela hospitalaria en acudir precozmente al médico" (Izzo 1938).

Alrededor del 15\% de los niños hospitalizados se presentaba con una de las formas más temidas, cual era la laringitis obstructiva o croup $^{11,13}$. En esta afección intervenían mecanismos locales y tóxicos que provocaban sofocación y asfixia debido a edema glótico y laringoespasmos, a los que se sumaba la obstrucción por pseudomembranas densas y adherentes que se asemejaban a una "tira de cuero" (en griego diphteria significa cuero o piel) $)^{14,15}$.

La traqueotomía fue propuesta a principios del siglo XIX por Bretonneau y su discípulo Trousseau. Hacia 1880 Bouchut y O'Dwyer separadamente impulsaron el uso de una vía más fisiológica "per viales naturales", al introducir del tubo de traqueostomía a través de la boca ${ }^{16}$. Durante los siguientes 50 años, hubo gran debate entre los círculos científicos norteamericanos y europeos en apoyo de uno $\mathrm{u}$ otro procedimiento, reportando distintas tasas de éxito. Tras años de perfeccionamiento en las técnicas e insumos, triunfó la intubación, reservándose la traqueostomía como medida excepcional ${ }^{7}$.

En nuestro país las primeras intubaciones en niños se hicieron los años $30^{6}$, cuando el debate mundial ya estaba prácticamente zanjado; según Izzo, durante aquel período "Los únicos adelantos que se han hecho se refieren sólo a los tubos y se han modificado en forma, tamaño, material de construcción, etc., para que se adapte lo mejor posible a la laringe, se eviten las úlceras de decúbito y no se obstruya tan fácilmente con las membranas"8.

En sus inicios, el laringoscopio era reemplazado por el dedo índice del operador, "Si la intubación se practica de noche, la iluminación toma un papel secundario, desde la más irradiante ampolleta hasta el más humilde cabo de vela sirven igualmente; pues la maniobra es totalmente dirigida por el tacto"; la confirmación que el tubo estaba bien instalado era básicamente clínica, si el niño podía hablar era una excelente señal que estaba en el lugar equivocado. La sedación y analgesia eran alternativas remotas $\mathrm{y}$, para la inmovilización parecía suficiente envolver al niño con una buena sábana. Se discutía el uso de la novocaína como anestésico local en mayores de 3 años, especialmente en los "neurópatas... con reflejos exagerados y que provocan arcadas... Por lo demás, si no se tiene a mano, puede lisa y llanamente suprimirse su aplicación aún en estos últimos casos".

Entre las posibles complicaciones de la intubación se describía la deglución del tubo metálico, el que era eliminado en las deposiciones. "La caída del tubo al esófago es un pequeño incidente sin mayor importancia”. Otra complicación temida era que el niño presente acostumbramiento al tubo, o quede "tubado", inconveniente no reportado en la estadística de Izzo, como tampoco ocurrieron infecciones. Las extubaciones accidentales y las reintubaciones eran bastante frecuentes y no suponían un verdadero problema ${ }^{8}$.

Más de la mitad de los niños afectados por el crup tenían indicación de instrumentalización de la vía aérea hasta superar la fase aguda de la enfermedad, período que fue acortado 
gracias al efecto de las antitoxinas ${ }^{11}$. El año 1934 la duración óptima de la intubación era de "entre las 56 y 60 horas, tiempo que parece ser el indispensable para que las lesiones se resuelvan" (Fernández Lagos 1934) ${ }^{6}$. Según Izzo "Este número de horas que es el que recomendamos, puede sin embargo, prolongarse en algunos casos excepcionales de abundantes membranas que no se hubieran eliminado suficientemente" 8 .

A pesar de los beneficios indiscutibles de la intubación, debemos considerar que la técnica consistía sólo en la introducción de un tubo que comunicaba la faringe con la tráquea, sin apoyo con ventilación con presión positiva, lo que generaba una sobrecarga respiratoria importante, pero sin duda menor que la provocada por respirar con la glotis obstruida. El año 1954, es decir casi 20 años después, Menghello publicaba que los cuidados adecuados de los pacientes intubados o traqueostomizados eran "humedad y calor suficientes, así como prácticas de enfermería necesarias (aspiración de secreciones, oxigenación, sedación)" "11. Faltarían aún varios años para que se instalaran los primeros ventiladores mecánicos y los cuidados intensivos pediátricos en nuestro país.

Si bien la mortalidad por crup diftérico descendió en forma importante gracias al descubrimiento de la antitoxina, posterior introducción de antibióticos y manejo de la vía aérea $^{15,17}$, en los años 40 y principios de los 50 aún fallecía cerca del $20 \%$ de los niños hospitalizados por esta afección ${ }^{3,11}$. Fue gracias a la vacunación universal, implementada en Chile el año 1955, que se logró el control de esta temida enfermedad, la que actualmente forma parte de la historia de la pediatría ${ }^{10}$.

\section{Referencias}

1.- Jiménez de la Jara J: Angelitos Salvados. Uqbar Editores 2009.

2.- Baeza Goñi A, Schonhaut L: Hospitalización del lactante. Rev Chil Pediatr 2012; 83 (3): 290-4.

3.- Romero H, Borgoño JM, Cervantes M, et al: Aportes a la epidemiologia de la difteria. Rev Chil Pediatr 1952;
23 (7): 277-89.

4.- Starr J: An Account of the Morbus Strangulatorius, Communicated in a Letter from John Starr, M. D. to C. Mortimer, M. D. Secret. R. S. \&c. Phil Trans R Soc 1749 46: 435-46. [citado 01-04-2013]. Disponible en http://rstl.royalsocietypublishing.org/content/46/491-496/435.full.pdf+html

5.- Laval E: Difteria en Chile. Rev Chil Infectol 2003; 20 (suppl): 42 .

6.- Fernández Lagos A: Intubación laríngea. Rev Chil Pediatr 1934; 413-7.

7.- Hardy A: Tracheotomy versus intubation: surgical intervention in diphtheria in Europe and the United States, 1825-1930. Bull Hist Med 1992; 66: 536-59.

8.- Izzo C: La intubación en el croup diftérico en el niño. Rev Chil Pediatr 1935; 241-54.

9.- Martínez P: Actualización de la situación epidemiológica de Difteria, Tétanos y Tétanos neonatal. El Vigía 2007; 24. [citado 10-02-13] Disponible en http://epi. minsal.cl/epi/html/elvigia/Vigia24.pdf

10.- Mobarec J: Contribución a la medicina preventiva de la difteria en Chile. Rev Chil Pediatr 1935; 30-7.

11.- Meneghello J, Undurraga O, Sepúlveda $H$ : Laringitis Diftérica. Rev Chil Pediatr 1954; 25 (4): 155-60.

12.- Rizzardini $M$, Undurraga O: Difteria maligna. Rev Chil Pediatr 1956; 27 (6): 242-8.

13.- Wiederhold A, Nazer J, Duffau G, Moreno B, Vallejos $E$ : Consideraciones sobre la clínica y la electrocardiografía de la difteria. Rev Chil Pediatr 1954; 25 (8): 3559.

14.- Buchanan G: Tracheotomy in diphtheria and croup. The British Medical Journal 1880. [citado 10-02-13] Disponible en: http://www.ncbi.nlm.nih.gov/pmc/articles/ PMC2240127/pdf/brmedj04877-0016.pdf

15.- Tuells J: La difteria, un camino hacia la sueroterapia y las anatoxinas. Revista Vacunas 2006; 7(1): 43-46. [citado 30-03-13] Disponible en: http://www.vacunas. org/index.php?option=com_content $\&$ task $=$ view\&id=7 11\&Itemid=329.

16.- Macewen $W$ : Introduction of tracheal tubes by the mouth instead of performing tracheotomy or laryngotomy. The British Medical Journal 1880. [citado 10-0213] Disponible en: http://www.ncbi.nlm.nih.gov/pmc/ articles/PMC2241154/pdf/brmedj04892-0008.pdf

17.- Opinel A, Tröhler U, Gluud C, et al: Commentary: The evolution of methods to assess the effects of treatments, illustrated by the development of treatments for diphtheria, 1825-1918. International Journal of Epidemiology 2011; 1-15. 